\title{
Thyroid Storm Masked by Hemodialysis and Glucocorticoid Therapy in a Patient with Rheumatoid Arthritis
}

\author{
Yohei Sasaki Yoshio Shimizu Junichiro Nakata \\ Toshiaki Kameda Masahiro Muto Isao Ohsawa \\ Hiroaki lo Chieko Hamada Satoshi Horikoshi \\ Yasuhiko Tomino
}

Division of Nephrology, Department of Internal Medicine, Juntendo University

Faculty of Medicine, Tokyo, Japan

\section{Key Words}

Thyroid storm · Graves' disease - Hemodialysis · Glucocorticoid therapy

\section{Abstract}

Thyroid function test values are generally at low levels in patients with end-stage kidney disease. Life-threatening thyrotoxicosis or thyroid storm is rare, especially in hemodialysis (HD) patients, and is characterized by multisystem involvement and a high mortality rate if not immediately recognized and treated. Here, we report a female patient with severe symptomatic thyroid storm, receiving long-term $\mathrm{HD}$ and glucocorticoid therapy. Methimazole at a dose of $15 \mathrm{mg}$ per day, $\beta$-adrenergic blockade and HD succeeded in controlling the patient's condition by gradually adjusting the target dry weight for hyperthyroidism-induced weight loss. When she was discharged from the hospital, her dry weight was reduced from 47.2 to $39.2 \mathrm{~kg}$. The management of patients with severe symptomatic thyroid storm on HD represents a rare scenario. It is essential to initiate the available treatments as early as possible to reduce its mortality.

\section{Introduction}

It has been reported that hypothyroidism may occur in $23.1 \%$ of stage 4 chronic kidney disease (CKD) patients [1], and ranges from 0 to $9.5 \%$ in end-stage kidney 
disease (ESKD) patients [2]. In general, thyroid function test values are low in patients with ESKD. Life-threatening thyrotoxicosis or thyroid storm is rare, especially in hemodialysis (HD) patients, and is characterized by multisystem involvement and a high mortality rate if not immediately recognized and treated $[3,4]$.

A multimodality treatment approach to patients with thyroid storm should be used, including $\beta$-adrenergic blockade, antithyroid drug therapy, inorganic iodine, corticosteroid therapy, volume resuscitation, and respiratory support and monitoring in an intensive care unit [3]. We report a case of severe symptomatic thyroid storm in a patient receiving long-term HD and glucocorticoid therapy.

\section{Case Report}

A 56-year-old female Japanese patient with diabetic nephropathy had been on maintenance HD three times a week since January 2006. She was diagnosed with rheumatoid arthritis (RA) and was taking prednisolone at a dose of $4 \mathrm{mg}$ per day. In August 2010, she began complaining of dyspnea, nausea, and fever. She was admitted to Juntendo University Hospital. One month earlier, she had had severe high blood pressure $(180 / 78 \mathrm{~mm} \mathrm{Hg})$, tachycardia $(110 / \mathrm{min})$, and cardiomegaly. The nephrologist made a presumptive diagnosis of congestive heart failure, and suggested she lose body weight gradually. She was given $60 \mathrm{mg}$ propranolol daily for sinus tachycardia. On physical examination, her blood pressure was $200 / 58 \mathrm{~mm} \mathrm{Hg}$, pulse 148 beats/min and regular, and temperature $38.2^{\circ} \mathrm{C}$. Her oxygen saturation in room air was $96 \%$. She had fine-finger tremor and a slightly enlarged thyroid gland without palpable nodules, which are integumentary changes suggestive of hyperthyroidism. There was no evidence of thyroid ophthalmopathy. The heart examination showed S3 on apex and a grade $2 / 6$ systolic murmur, and coarse crackles were present in both lungs, with slight peripheral edema. She also had symmetric synovitis of the wrists, ankles, and carpometacarpal joints. Findings from other physical examinations were normal.

A chest X-ray showed cardiomegaly (cardiothoracic ratio 58\%) with pulmonary congestion. The 12-lead ECG on admission indicated sinus tachycardia. Laboratory data on admission were compatible with chronic renal failure receiving HD (serum creatinine $5.18 \mathrm{mg} / \mathrm{dl}$; urea nitrogen 66 $\mathrm{mg} / \mathrm{dl}$ ). A blood test showed hemoglobin of $11.0 \mathrm{~g} / \mathrm{dl}$, hematocrit $35.1 \%$ and white blood cell count $9,700 / \mu \mathrm{l}$. A thyroid function test revealed a TSH level of $<0.01 \mu \mathrm{IU} / \mathrm{ml}$ (normal $0.5-5$ ), FT4 level $>7.8$ $\mathrm{ng} / \mathrm{dl}$ (normal 0.9-1.7), FT3 level $11.4 \mathrm{pg} / \mathrm{ml}$ (normal 2.3-4.3), and TSH receptor antibody $10.9 \mathrm{IU} / \mathrm{l}$ (normal <1). On the basis of the Burch-Wartofsky score [4], which is widely used as a global scale for the evaluation of thyrotoxicosis, the patient's condition was diagnosed as thyroid storm due to Graves' disease [thyrotoxicosis with hyperthermia (10 points), agitation (10 points), gastrointestinal symptoms, nausea, vomiting (10 points), tachycardia ( 25 points), and moderate heart failure (10 points)]. A total score of 65 or more is highly suggestive of definite thyroid storm.

Fig. 1 shows the clinical course of the present case. Treatment was initiated with methimazole (MMI) at a dose of $15 \mathrm{mg}$ per day, and HD was performed. Her body weight was adjusted to improve the pulmonary congestion, hypertension, and level of atrial natriuretic peptide (ANP) for hyperthyroidism-induced weight loss. Target dry weight was decreased gradually, ranging from 0.2 to $1.0 \mathrm{~kg}$ (median 0.4 ) at each dialysis. A few days later, agitation and gastrointestinal symptoms disappeared while her plasma level of ANP decreased from 202 to $100 \mathrm{pg} / \mathrm{ml}$; we continued to reduce her target dry weight. The patient's FT4 level decreased to $2.6 \mathrm{ng} / \mathrm{dl}$ when titration of the MMI dose was started. Propranolol was maintained for control of hypertension and sinus tachycardia, and the same dosage of glucocorticoid as before admission was also continued. Since the thyroid function parameters were normalized, she was discharged from the hospital and her dry weight was finally reduced from 47.2 to $39.2 \mathrm{~kg}$. Approximately 1 year after discharge, she is well and without any subjective symptoms. 


\section{Discussion}

It has been reported that hypothyroidism may occur in $23.1 \%$ of stage 4 CKD patients [1], and ranges from 0 to $9.5 \%$ in ESKD patients [2]. In general, thyroid function tests show low levels in patients with ESKD. Life-threatening thyrotoxicosis or thyroid storm is rare, especially in HD patients, and is characterized by multisystem involvement and a high mortality rate if not immediately recognized and treated [3]. The diagnosis of thyroid storm was determined on the basis of the Burch-Wartofsky score, which is widely used for evaluation of the severity of thyrotoxicosis [4, 5]. In this case, clinical findings showed a total score of 65 or more, which is highly suggestive of definite thyroid storm.

There were two predominant features in this case. One was maintenance HD for 4 years. CKD affects both the hypothalamus-pituitary-thyroid axis and peripheral metabolism of thyroid hormones [6, 7]. Serum thyrotropin concentrations are usually normal or elevated, and free and total T3 and T4 concentrations are normal or low in patients with CKD [6]. It is possible that some of these patients had impaired renal handling of iodine, resulting in elevation of serum iodine levels with prolongation of the Wolff-Chaikoff effect $[7,8]$. Since our patient was confirmed as euthyroid 5 months before admission, her thyroid function might have risen gradually over several months. The other feature was that she had been prescribed prednisolone for several years for the treatment of RA by a rheumatologist. Glucocorticoid agents (e.g. prednisone or dexamethasone) inhibit T4-to-T3 conversion, and the effect of the agent enables rapid reduction of serum T3 in patients with thyroid storm or severe cardiac disease [9]. Thus, a variety of signs and symptoms characterized by thyrotoxicosis was probably masked by long-term HD and glucocorticoid therapy.

RA and Graves' disease are two common autoimmune diseases with similar prevalence in the general population and both of them affect predominantly middleaged women [10]. There is no direct evidence implicating common genetic factors. Recently, a possible common pathogenic mechanism in Graves' disease and RA involving an insulin-like growth factor receptor-1 (IGF1-R) on fibroblasts, a pathway that mediates inflammatory responses, was reported [11]. Moreover, it was suggested that a functional variant of the FCRL3 gene, encoding Fc receptor-like 3, is associated with RA and several autoimmune diseases, including Graves' disease [12].

A multimodality treatment approach should be used in patients with thyroid storm, including $\beta$-adrenergic blockade, antithyroid drug therapy, inorganic iodine, corticosteroid therapy, volume resuscitation, and if necessary, respiratory support and monitoring in an intensive care unit [3]. The present case was treated using oral prednisolone, a reduced dose of MMI and $\beta$-adrenergic blockade, with careful consideration of her body weight, loss of kidney function, drug removal by dialysis, and accumulation of the drug in the body. The best therapy for hyperthyroidism in HD patients remains a matter of debate [9]. Antithyroid drugs, specifically thionamides (either MMI or propylthiouracil), are commonly used and primarily interfere with thyroid hormone synthesis [9]. The use of antithyroid drugs as initial treatment varies according to geographic location; they are used in the majority of patients in Europe and Asia, but radioiodine is used more often than medication in the United States [13, 14]. The superiority of either MMI or propylthiouracil is not clearly established; however, MMI has a longer intrathyroidal half-life, often allowing for once-daily dosing, 
and some studies have shown that it has greater efficacy and fewer side effects $[9,13$, 15]. This case illustrates successful management by initiation of MMI and $\beta$-adrenergic blockade in a patient with thyroid storm on HD.

\section{Conclusion}

In conclusion, the management of patients with severe symptomatic thyroid storm on HD represents a challenge for nephrologists. We should recognize and treat such patients immediately, together with endocrinologists and critical care physicians, using appropriate treatment modalities in order to avoid a catastrophic outcome.

\section{Disclosure Statement}

The authors have nothing to declare.

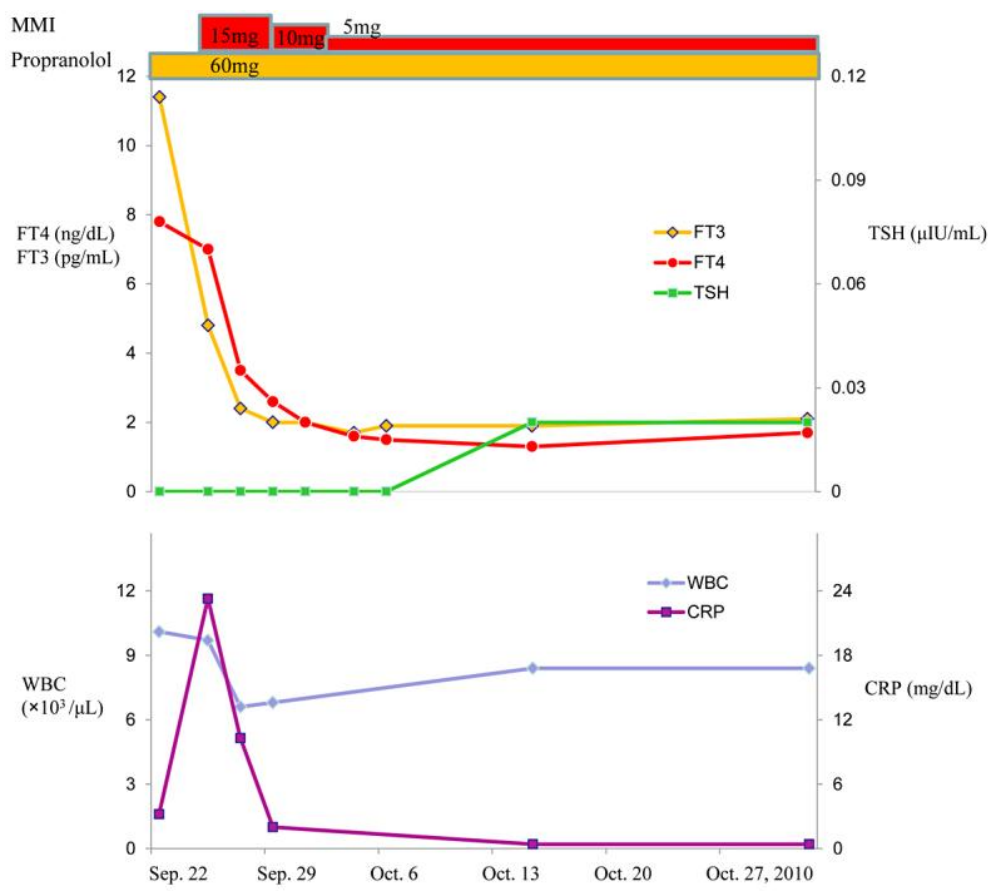

Fig. 1. Clinical course of the present case. The patient was diagnosed with thyroid storm due to Graves' disease. Treatment was initiated with MMI at a dose of $15 \mathrm{mg}$ per day and her target dry weight was adjusted for hyperthyroidism-induced weight loss. A few days after admission, agitation and gastrointestinal symptoms disappeared, and titration of the MMI dose was started. 


\section{References}

1 Lo JC, Chertow GM, Go AS, Hsu CY: Increased prevalence of subclinical and clinical hypothyroidism in persons with chronic kidney disease. Kidney Int 2005;67:1047-1052.

-2 Kaptein EM, Quion-Verde H, Chooljian CJ, Tang WW, Friedman PE, Rodriquez HJ, Massry SG: The thyroid in end-stage renal disease. Medicine 1988;67:187-197.

-3 Bahn Chair RS, Burch HB, Cooper DS, Garber JR, Greenlee MC, Klein I, Laurberg P, McDougall IR, Montori VM, Rivkees SA, Ross DS, Sosa JA, Stan MN: Hyperthyroidism and other causes of thyrotoxicosis: management guidelines of the American Thyroid Association and American Association of Clinical Endocrinologists. Thyroid 2011;21:593-646.

4 Burch HB, Wartofsky L: Life-threatening thyrotoxicosis. Thyroid storm. Endocrinol Metab Clin North Am 1993;22:263-277.

5 Yoshino T, Kawano D, Azuhata T, Kuwana T, Kogawa R, Sakurai A, Tanjoh K, Yanagawa T: A patient with Graves' disease who survived despite developing thyroid storm and lactic acidosis. Ups J Med Sci 2010;4:282-286.

6 Kaptain EM: Thyroid hormone metabolism and thyroid diseases in chronic renal failure. Endocr Rev 1996;17:45-63.

77 Iglesias P, Díez JJ: Thyroid dysfunction and kidney disease. Eur J Endocrinol 2009;160:503-515.

8 Bando Y, Ushiogi Y, Okafuji K, Toya D, Tanaka N, Miura S: Non-autoimmune primary hypothyroidism in diabetic and non-diabetic chronic renal dysfunction. Exp Clin Endocrinol Diabetes 2002;110:408-415.

9 Brent GA: Clinical practice. Graves' disease. N Engl J Med 2008;358:2594-2605.

10 Bourikas LA, Sidiropoulos PI, Goulielmos GN, Boumpas DT, Kritikos HD: Concomitant onset of Graves' disease and rheumatoid arthritis after a serious life event. J Clin Rhe umatol 2006;12:326-327.

-11 Pritchard J, Tsui S, Horst N, Cruikshank WW, Smith TJ: Synovial fibroblasts from patients with rheumatoid arthritis, like fibroblasts from Graves' disease, express high levels of IL-16 when treated with Igs against insulin-like growth factor-1 receptor. J Immunol 2004;173:3564-3569.

12 Kochi Y, Yamada R, Suzuki A, Harley JB, Shirasawa S, Sawada T, Bae SC, Tokuhiro S, Chang X, Sekine A, Takahashi A, Tsunoda T, Ohnishi Y, Kaufman KM, Kang CP, Kang C, Otsubo S, Yumura W, Mimori A, Koike T, Nakamura Y, Sasazuki T, Yamamoto K: A functional variant in FCRL3, encoding Fc receptor-like 3, is associated with rheumatoid arthritis and several autoimmunities. Nat Genet 2005;37:478-485.

13 Cooper DS: Antithyroid drugs. N Engl J Med 2005;352:905-917.

14 Wartofsky L, Glinoer D, Solomon B, Nagataki S, Lagasse R, Nagayama Y, Izumi M: Differences and similarities in the diagnosis and treatment of Graves' disease in Europe, Japan, and the United States. Thyroid 1991;1:129-135.

15 Nakamura H, Noh JY, Itoh K, Fukata S, Miyauchi A, Hamada N: Comparison of methimazole and propylthiouracil in patients with hyperthyroidism caused by Graves' disease. J Clin Endocrinol Metab 2007;92:2157-2162. 